\title{
Compatibilidade de inseticidas utilizados na cultura do eucalipto com Metarhizium rileyi (Farlow) (=Nomuraea rileyi)
}

\author{
Maiara Amaral da Costa ${ }^{1}$, Elisângela de Souza Loureiro ${ }^{1}$, Luis Gustavo Amorim Pessoa ${ }^{1}$, \\ Pamella Mingotti Dias ${ }^{2}$
}

\author{
${ }^{1}$ Universidade Federal de Mato Grosso do Sul, Campus de Chapadão do Sul, Chapadão do Sul, Mato Grosso do Sul, Brasil. E-mail: \\ maiara_cr@hotmail.com, elisangela.loureiro@ufms.br, luis.pessoa@ufms.br \\ ${ }^{2}$ Universidade Federal da Grande Dourados, Dourados, Mato Grosso do Sul, Brasil. E-mail: pamellamingotti@ hotmail.com
}

Recebido: 04/06/2017; Aceito: 05/09/2017

\section{RESUMO}

Objetivou-se avaliar a compatibilidade dos inseticidas químicos (Orthene ${ }^{\circledR} 750$ BR, Mospilan ${ }^{\circledR}$, Imidacloprid ${ }^{\circledR}$ NORTOX e Evidence $\left.{ }^{\circledR} 700 \mathrm{WG}\right)$, sobre o desenvolvimento de Metarhizium rileyi. Os experimentos foram realizados "in vitro", avaliando os efeitos sobre o crescimento vegetativo, produção de conídios (esporulação) e germinação do fungo M. rileyi (isolado UFMS 03) na presença de diferentes inseticidas. Após a obtenção dos dados de diâmetro de colônias, produção de conídios e germinação foram calculados o fator de compatibilidade. Os inseticidas Mospilan ${ }^{\circledR}$, Imidacloprid ${ }^{\circledR}$ NORTOX e Evidence ${ }^{\circledR} 700$ WG proporcionaram crescimento vegetativo semelhante a testemunha, considerados compatíveis com M. rileyi enquanto que Orthene ${ }^{\circledR} 750$ BR foi considerado moderadamente tóxico. Os inseticidas Imidacloprid ${ }^{\circledR}$ NORTOX e Evidence ${ }^{\circledR} 700$ WG não interferiram na produção de conídios proporcionando valores semelhantes a testemunha. Não houve efeito dos tratamentos sobre a germinação dos conídios de $M$. rileyi.

Palavras-chave: fungo entomopatogênico, controle biológico, controle químico, pragas florestais

\section{Compatibility of insecticides used in the eucalyptus crop with Metarhizumi rileyi (Farlow) (=Nomuraea rileyi)}

\begin{abstract}
The aim of this study was to evaluate the compatibility of chemical insecticides (Orthene ${ }^{\circledR} 750 \mathrm{BR}$, Mospilan ${ }^{\circledR}$, Imidacloprid $^{\circledR}$ NORTOX and Evidence ${ }^{\circledR} 700$ WG) on the development of Metarhizium rileyi. The experiments were carried out "in vitro", evaluating the effects on vegetative growth, conidia (sporulation) and germination of the fungus M. rileyi (isolated UFMS 03) in the presence of different insecticides. After obtaining the data of colonies diameter, conidia production and germination, the compatibility factor was calculated. The insecticides Mospilan $^{\circledR}$, Imidacloprid ${ }^{\circledR}$ NORTOX and Evidence ${ }^{\circledR} 700$ WG provided vegetative growth similar to control, considered compatible with M. rileyi while Orthene ${ }^{\circledR} 750$ BR was considered moderately toxic. The insecticides Imidacloprid $^{\circledR}$ NORTOX and Evidence ${ }^{\circledR} 700 \mathrm{WG}$ did not interfere in the production of conidia giving similar values to the control. There was no effect of the treatments on the germination of M. rileyi conidia.
\end{abstract}

Keywords: entomopathogenic fungus, biological control, chemical control, forest pests 


\section{Introdução}

As áreas reflorestadas com espécies arbóreas para fins comerciais somam no Brasil mais de 7,7 milhões de hectares, sendo que apenas a cultura de eucalipto é responsável por $73 \%$ deste total (Souza et al., 2016). O crescimento da área cultivada com eucalipto causa severa queda na biodiversidade, essa falta de competição favorece a proliferação de insetos fitófagos e doenças. Plantações de Eucalyptus permitem uma adaptação vantajosa de insetos, devido à disponibilidade de fonte alimentar e à fragilidade desse novo ecossistema, dificultando o desenvolvimento dos inimigos naturais da praga (Pereira, 2007).

As perdas de produtividade no setor florestal brasileiro estão relacionadas a introdução de pragas exóticas como o psilídeo-de-concha Glycaspis brimblecombei (Hemiptera: Psyllidae) (Hemiptera: Psyllidae), o percevejo-bronzeado Thaumastocoris peregrinus (Hemiptera: Thaumastocoridae) e a vespada-galha Leptocybe invasa (Hymenoptera: Eulophidae) (Wilcken, 2008).

As florestas de eucalipto sofrem perdas também com as pragas nativas e têm como principais problemas, as formigas cortadeiras, cupins, lagartas desfolhadoras e o besouro amarelo. No Brasil, existem dez espécies de lagartas desfolhadoras, destacando-se por maior ocorrência no campo a lagarta parda do eucalipto Thyrinteina arnobia (Lepidoptera: Geometridae) (Pastori et al., 2012).

Segundo Oliveira et al. (2011), o controle da lagarta parda em cultivos florestais, em alguns casos utiliza-se inseticidas químicos. Porém, a aplicação destes produtos torna-se complexa devido a grande extensão das áreas de plantio e à altura das plantas.

O controle biológico natural com microrganismos é um método muito viável e eficaz para o manejo integrado de pragas florestais. O fungo Metarhizium rileyi (Ascomycota: Clavicipitaceae) (Kepler et al., 2014), é um entomopatógeno cosmopolita que ocorre infectando lepidópteros praga de diversas culturas de importância econômica em várias regiões do mundo (Costa et al., 2015), com possibilidades de aplicação em lagartas desfolhadoras em florestas.

De maneira pratica e sustentável, a preservação dos fungos entomopatogênicos no ambiente é resultado da utilização de produtos químicos seletivos, promovendo a eficaz ação de inimigos naturais. De acordo com Silva et al. (2005) as interações entre fungos entomopatogênicos e produtos fitossanitários, podem ser considerados positivas, em casos de sinergismo entre o patógeno e o produto utilizado, ou ainda, negativo caso ocorra a inibição de um dos componentes do patógeno.

O presente trabalho teve como objetivo avaliar se os inseticidas químicos (Orthene ${ }^{\circledR} 750$ BR, Mospilan ${ }^{\circledR}$, Imidacloprid $^{\circledR} \quad$ NORTOX e Evidence ${ }^{\circledR} \quad 700$ WG) utilizados no manejo de pragas exóticas do eucalipto (psilídeo-de-concha, o percevejo bronzeado e a vespada-galha), afetam o desenvolvimento de $M$. rileyi que apresenta potencial para o manejo da lagarta parda do eucalipto T. arnobia, permitindo assim o controle de pragas exóticas e nativas em conjunto.

\section{Material e Métodos}

Os experimentos foram realizados "in vitro", onde foram avaliados efeitos sobre o crescimento vegetativo, germinação e produção de conídios (esporulação) do fungo $M$. rileyi (isolado UFMS 03) na presença de diferentes inseticidas.

Foram utilizados os produtos químicos Orthene ${ }^{\circledR} 750$ BR, Mospilan ${ }^{\circledR}$, Imidacloprid ${ }^{\circledR}$ NORTOX e Evidence ${ }^{\circledR}$ 700 WG, em dose média, de acordo com as especificações dos fabricantes, com volume de calda fixo de $100 \mathrm{~L} \mathrm{ha}^{-1}$ (Tabela 1). O tratamento testemunha consistiu apenas do meio de cultura batata-dextrose-ágar (BDA) sem adição de inseticidas.

Para que as avaliações de crescimento vegetativo e produção de conídios de $M$. rileyi fossem efetuadas, os inseticidas foram acrescentados a $200 \mathrm{~mL}$ de BDA não solidificado. Após agitação, foram vertidos em placas de Petri com $9 \mathrm{~cm}$ de diâmetro e identificadas de acordo com cada tratamento. Após ocorrer a solidificação do meio, realizou-se a inoculação do fungo $M$. rileyi com o auxílio da agulha de platina em três pontos equidistantes. As placas foram embaladas com plástico filme PVC e acondicionadas em câmara climatizada do tipo B.O.D a temperatura de $25 \pm 1{ }^{\circ} \mathrm{C}$, umidade relativa de $70 \pm 10 \%$ e fotofase de 12 horas durante 12 dias, para promover o crescimento das colônias.

Tabela 1. Inseticidas utilizados em Eucalipto recomendados para o manejo de pragas exóticas.

\begin{tabular}{lllll}
\hline Produto Comercial & Nome técnico & Grupo químico & Classe $^{1}$ & Dose $^{2}$ \\
\hline Orthene $^{\circledR} 750 \mathrm{BR}$ & Acefato & Organofosfarado & $\mathrm{I} / \mathrm{A}$ & $400 \mathrm{~g} \mathrm{ha}^{-1}$ \\
Mospilan $^{\circledR}$ & Acetamiprido & Neonecotinoide & $\mathrm{I}$ & $250 \mathrm{~g} \mathrm{ha}^{-1}$ \\
Imidacloprid $^{\circledR}$ NORTOX & Imidacloprid & Neonecotinoide & $\mathrm{I}$ & $150 \mathrm{~g} \mathrm{ha}^{-1}$ \\
Evidence $^{\circledR} 700 \mathrm{WG}$ & Imidacloprido & Neonecotinoide & $\mathrm{I}$ & $450 \mathrm{~g} \mathrm{ha}^{-1}$ \\
\hline
\end{tabular}

${ }^{\mathrm{T}} \mathrm{I}$ = Inseticida; A = Acaricida. ${ }^{2}$ Dose média recomendada pelos fabricantes. 
O delineamento experimental foi inteiramente casualizado com cinco tratamentos (testemunha e inseticidas), cada um composto por quatro placas totalizando doze colônias. Dessas, apenas seis foram aleatoriamente apontadas, resultando assim em seis repetições por tratamento.

Para avaliação do crescimento vegetativo foram efetuadas medições das colônias em dois eixos ortogonais, obtendo-se o diâmetro médio de cada colônia, em centímetros. Nas análises da produção de conídios, as colônias foram retiradas das placas com auxílio de um bisturi cirúrgico estéril e transferidas para tubos de ensaio, contendo $10 \mathrm{~mL}$ de água destilada + $0,1 \%$ espalhante adesivo (Tween $80^{\circledR}$ ). Em seguida foi realizada a agitação no aparelho Vortex, para que houvesse a desagregação dos conídios. Após, realizouse uma diluição e a contagem de conídios foi realizada com o auxílio da câmara Neubauer, utilizando-se microscópio óptico (Alves et al., 2008).

Os dados de crescimento vegetativo e de produção de conídios foram submetidos ao teste de Tukey a 5\% de probabilidade, os dados de crescimento vegetativo foram originais e os dados de produção de conídios foram transformados para $(x+0,5)^{0,5}$, respectivamente.

Para a avaliação da germinação foi utilizada uma suspensão de $1,0 \times 108$ conídios $\mathrm{mL}^{-1}$ de $M$. rileyi, a qual foi mantida em repouso por duas horas em caldas contendo os diversos inseticidas nas dosagens recomendadas (Tabela 1). Após duas horas, foi colocada 1,0 mL da suspensão com auxílio de uma pipeta graduada em placas de Petri e com a alça Drigalsky espalhadas sobre o meio de cultura BDA. Após a inoculação, as placas foram identificadas, embaladas com filme PVC e colocadas por 20 horas em câmara climatizada tipo B.O.D a $25 \pm 1^{\circ} \mathrm{C}$, umidade relativa de $70 \pm 10 \%$ e fotofase de 12 horas (Alves et al., 2008).

$\mathrm{O}$ delineamento experimental foi inteiramente casualizado com cinco tratamentos (testemunha e os inseticidas), e a repetição composta por quatro placas de Petri (dividida em quatro quadrantes). Os dados de germinação foram submetidos ao teste Tukey $(p=0,05)$.
Após a incubação, as placas foram divididas em quatro quadrantes sendo contados 100 conídios em cada quadrante, estabelecendo posteriormente a porcentagem de germinação. $\mathrm{Na}$ análise dos resultados de germinação, foi adotado o padrão do Laboratório de Controle Biológico do Instituto Biológico de Campinas: germinação alta $80-100 \%$, germinação médio-alta 60 $79 \%$, germinação média 50-59\%, germinação médiobaixa $30-49 \%$ e germinação baixa 0-29\% (Zappelini et al., 2005).

A partir dos dados foram calculados o fator de compatibilidade (IB = índice biológico), proposto por Rossi-Zalaf et al. (2008) que classifica os produtos quanto a sua toxidade observando os efeitos em relação aos parâmetros avaliados (crescimento vegetativo, produção de conídios e germinação). De acordo com os valores obtidos, foi realizada comparação com os limites estabelecidos para determinação da toxicidade dos produtos estudados, como segue: 0 a 41 - tóxico; 42 a 66 - moderadamente tóxico; > 66 - compatível.

\section{Resultados e Discussão}

Os inseticidas Evidence ${ }^{\circledR} 700 \mathrm{WG}$, Imidacloprid ${ }^{\circledR}$ NORTOX, Mospilan ${ }^{\circledR}$ apresentaram crescimento vegetativo semelhante à testemunha $(2,79 ; 2,84 ; 2,42 \mathrm{e}$ $2,88 \mathrm{~cm}$, respectivamente), não havendo efeito inibitório dos produtos. O inseticida/acaricida Orthene ${ }^{\circledR} 75$ BR proporcionou o menor crescimento $(2,08 \mathrm{~cm})$, diferindo estatisticamente da testemunha, porém não diferindo do Mospilan $^{\circledR}$, observando que os produtos interferiram de certa forma no crescimento do fungo (Tabela 2).

Ao avaliar a conidiogênese observou-se que os inseticidas Evidence $^{\circledR} 700$ WG e Imidacloprid $^{\circledR}$ NORTOX foram semelhantes à testemunha $(2,09 ; 2,28$ e $2,37 \times 10^{9}$ con $\mathrm{mL}^{-1}$ ), porém não diferindo de Mospilan $^{\circledR}\left(1,72 \times 10^{9}{\left.\text { con } \mathrm{mL}^{-1}\right) \text {. O inseticida Mospilan }}^{\circledR}\right.$ e o inseticida/acaricida Orthene ${ }^{\circledR} 750$ BR apresentaram produção de conídios inferior à testemunha $(1,72$; $1,6 \times 10^{9}$ con $\mathrm{mL}^{-1}$, respectivamente) (Tabela 2 ).

Tabela 2. Diâmetro médio das colônias $(\mathrm{cm})$ e número médio de conídios $(\mathrm{X} \pm \mathrm{EP})$ e média da germinação $(\%)$ produzidos por colônia de $M$. rileyi $(\mathrm{n}=5)$ na presença de inseticidas.

\begin{tabular}{|c|c|c|c|}
\hline Tratamento & Diâmetro $(\mathrm{cm})^{1}$ & Conídios $\left(\mathrm{x} 10^{9}\right)^{1,2}$ & Germinação ${ }^{1,3}$ \\
\hline Testemunha & $2,88 \pm 0,22 \mathrm{a}$ & $2,37 \pm 1,13 \mathrm{a}$ & 100 a (alta) \\
\hline Orthene ${ }^{\circledR} 750$ BR & $2,08 \pm 0,19 b$ & $1,60 \pm 0,36 \mathrm{c}$ & 100 a (alta) \\
\hline Mospilan $^{\circledR}$ & $2,42 \pm 0,29 a b$ & $1,72 \pm 0,38 \mathrm{bc}$ & 100 a (alta) \\
\hline Imidacloprid $^{\circledR}$ NORTOX & $2,84 \pm 0,35 \mathrm{a}$ & $2,28 \pm 0,58 a b$ & 100 a (alta) \\
\hline Evidence $^{\circledR} 700 \mathrm{WG}$ & $2,79 \pm 0,11 \mathrm{a}$ & $2,09 \pm 0,34 \mathrm{abc}$ & 100 a (alta) \\
\hline $\mathrm{CV}(\%)$ & 11,52 & 16,79 & 0,0 \\
\hline
\end{tabular}


Avaliando a compatibilidade dos fungos entomopatogênicos Beauveria bassiana, M. rileyi, Isaria fumosorosea, Isaria sinclairii e Lecanicillium muscarium em relação alguns inseticidas, entre esses o acefato (DUARTE et al., 2016), observaram redução na produção de conídios, crescimento vegetativo e na viabilidade das estruturas reprodutivas dos isolados quando estes estiveram em contato com as moléculas químicas do acefato.

Em estudo de compatibilidade realizado por SosaGomez (2005), Imidacloprid ${ }^{\circledR}$ foi seletivo em condições temporárias, após 4 horas de exposição, porém, em contato permanente inibiu o crescimento de $M$. rileyi. Esse autor relatou ainda que os produtos Klap ${ }^{\circledR}$, Dimilin $^{\circledR}$ e Actara $^{\circledR}$ são os menos nocivos, existindo assim a possibilidade do uso conjunto com M. rileyi, devido a sua compatibilidade. Os produtos utilizados por Sosa-Gomez (2005), não são os mesmos testados neste trabalho, porém vale ressaltar que o produto Actara ${ }^{\circledR}$ pertence ao mesmo grupo químico neonicotinoídes dos inseticidas Mospilan ${ }^{\circledR}$, Imidacloprid $^{\circledR}$ NORTOX e Evidence ${ }^{\circledR} 700$ WG.

Uma hipótese sustentada por Moino Júnior e Alves (1998) e que poderia explicar o crescimento vegetativo do fungo mesmo em contato com as moléculas químicas, é que o fungo metabolize as toxinas dos produtos ocorrendo liberação de moléculas que podem ser utilizadas como nutrientes secundários. Ou que o fungo utilize todo seu esforço reprodutivo, quando em contato com princípios tóxicos que prejudiquem seu desenvolvimento, promovendo assim o crescimento vegetativo, conidiogênese e germinação.

Os conídios de $M$. rileyi apresentaram $100 \%$ de germinação quando exposto aos tratamentos, sendo classificada como germinação alta (Zappelini et al., 2005). O fato de o crescimento vegetativo ser inibido não indicada a redução na esporulação ou da viabilidade dos conídios (Zimmermann, 1975). Como pode ser observado nesse estudo, onde o inseticida/acaricida Orthene $^{\circledR} 750$ BR apresentou redução no crescimento vegetativo e conidiogenese, porém não interferiu na germinação de $M$. rileyi.

Ao calcular o índice biológico utilizando os parâmetros avaliados, os inseticidas Mospilan ${ }^{\circledR}$, Imidacloprid $^{\circledR}$ NORTOX e Evidence ${ }^{\circledR} 700$ WG foram classificados como compatíveis com $M$. rileyi, enquanto o inseticida Orthene ${ }^{\circledR} 750 \mathrm{BR}$ foi classificado como moderadamente toxico ao fungo (Tabela 3 ).

Estes resultados confirmam os resultados apresentados por Duarte et al. (2016), os quais classificaram o ingrediente ativo acefato como moderadamente tóxico a quase todos os isolados de fungos entomopatogênicos testados, entre eles o $M$. rileyi isolado LCMAP101. Testes utilizando os produtos químicos: Actara mix (thiamethoxam + cipermetrina) e
Actara (thiametoxam) classificaram esses inseticidas do grupo dos neonecotinoides, como compatível a $M$. rileyi, pois não afetaram nenhum dos parâmetros biológicos, independente da concentração utilizada máxima e mínima (Batista Filho et al., 2001; Batista Filho et al., 2003).

Tabela 3. Classificação dos inseticidas de acordo com a sua toxicidade (valor “IB"), segundo Rossi-Zalaf et al. (2008)

\begin{tabular}{lcc}
\hline Tratamentos & $\begin{array}{c}\text { Índice } \\
\text { biológico (\%) }\end{array}$ & Classificação \\
\hline Orthene $^{\circledR} 750$ BR & 60,99 & Moderadamente tóxico \\
Mospilan $^{\circledR}$ & 69,74 & Compatível \\
Imidacloprid $^{\circledR}$ Nortox & 94,62 & Compatível \\
Evidence $^{\circledR} 700$ WG & 86,93 & Compatível \\
\hline
\end{tabular}

A vantagem dos estudos in vitro é maximizar a exposição do microrganismo à ação do produto químico, embora esse método não represente as condições de campo, e as múltiplas interações que existem nos ambientes naturais, espera-se que as informações geradas permitam inferir quais produtos podem interferir sobre as populações desses microrganismos (Moino Júnior; Alves, 1998).

Loureiro et al. (2002), relataram que os produtos Tiametoxam, Imidaclopride, Bifentrina e Acefato, diminuíram significativamente a produção de conídios do fungo Metarhizium anisopliae quando comparados aos da testemunha o que também ocorreu no presente trabalho com o produto Orthene ${ }^{\circledR} 750 \mathrm{BR}$ (acefato).

Neves et al. (2001) concluíram que o uso dos inseticidas Acetamiprid, Imidacloprid e Thiametoxam, não tem efeito negativo na germinação, crescimento vegetativo e produção de conídios de $B$. bassiana, $M$. anisopliae e Paecilomyces sp. Apesar de não se tratar do mesmo isolado ou espécie de fungo, os dados do presente trabalho concordam com a literatura onde os inseticidas Mospilan $^{\circledR}$, Imidacloprid ${ }^{\circledR} \quad$ NORTOX (Acetamiprid e Imidacloprid) não apresentaram efeitos negativos nos dados de germinação, crescimento vegetativo e produção de conídios do $M$. rileyi.

No contexto do MIP todas as táticas de controle são utilizadas e a estratégia de aplicação de fungos entomopatogênicos associado a inseticida, deve-se dar prioridade ao uso dos produtos que apresentam compatibilidade, levando em consideração que estes entomopatógenos podem adaptar-se ao ambiente, apresentando posteriormente ação de enzootia e epizootia contribuindo para o controle biológico natural. Em função dos resultados obtidos é possível concluir que os inseticidas Mospilan ${ }^{\circledR}$, Imidacloprid $^{\circledR}$ NORTOX e Evidence ${ }^{\circledR} 700$ WG são compatíveis com o fungo $M$. rileyi 


\section{Conclusões}

Os inseticidas Mospilan ${ }^{\circledR}$, Imidacloprid ${ }^{\circledR}$ NORTOX e Evidence ${ }^{\circledR} 700 \mathrm{WG}$ foram compatíveis com M. rileyi, porém destes, apenas o Mospilan ${ }^{\circledR}$ reduziu a produção de conídios.

O inseticida Orthene ${ }^{\circledR} 750$ BR foi moderadamente tóxico reduzindo significativamente o crescimento vegetativo e a produção de conídios

\section{Referências Bibliográficas}

Alves, S.B.; Lopes, R.B.; Vieira, S.A.; Tamai, M. A., 2008. Fungos entomopatogênicos usados no controle de pragas na América Latina, in: Alves, S.B.; Lopes, R.B. (Eds). Controle microbiano de pragas na América Latina. Piracicaba-SP: FEALQ, pp. 69-110.

Batista filho, A.; Almeida, J. E. M.; Lamas, C., 2001. Effect of thiamethoxam on entomopathogenic microorganisms. Neotropical Entomology, 30, 437-447.

Batista filho, A.; Ramiro, Z. A.; Almeida, J. E. M.; Leite, L. G.; Cintra, E. R.; Lamas, C., 2003. Manejo integrado de pragas em soja: impacto de inseticidas sobre inimigos naturais. Arquivos do Instituto Biológico,70, 61-67.

Costa, V. H. D.; Soares, M. A.; Rodríguez, F. A. D.; Zanuncio, J. C., Silva, I. M.; Valicente, F. H., 2015. Nomuraea rileyi (Hypocreales: Clavicipitaceae) in Helicoverpa armigera (Lepidoptera: Noctuidae) larvae in Brazil. Florida Entomologist, 98, 796-798.

Duarte, R.T.; Gonçalves, K. C.; Espinosa, D. J.; Moreira, L. F.; De Bortoli, S. A.; Humber, R. A.; Polanczyk, R., 2016. A. Potential of Entomopathogenic Fungi as Biological Control Agents of Diamondback Moth (Lepidoptera: Plutellidae) and Compatibility With Chemical Insecticides. Journal of Economic Entomology, 109, 594-601.

Kepler, R. M.; Humber, R. A.; Bischoff, J. F.; Rehner, S. A. 2014. Clarification of generic and species boundaries for Metarhizium and related fungi through multigene phylogenetics. Mycologia, 106, 811829.

Loureiro, E. D. S.; Moino Junior, A.; Arnosti, A.; Souza, G. C. D. 2002. Efeito de Produtos fitossanitários Químicos Utilizados em Alface Crisântemo Sobre Fungos Entomopatogênicos. Neotropical Entomology, 31, 263-269.

Moino Júnior, A. M.; Alves, S. B., 1998. Efeito de imidaclopid e fipronil sobre Beauveria bassiana e Metarhizium anisopliae e no comportamento de limpeza de Heterotermes tenuis (Hagen). Anais da Sociedade Entomológica Brasileira, 27, 661-619.
Neves, P. M. O. J.; Hirose, E.; Tchujo, P. T.; Moino Júnior, A., 2001. Compatibility of entomopathogenic fungi with neonicotinoids insecticides. Neotropical Entomology, 30, 263 268.

Oliveira, H. N.; Espindula, M. C.; Duarte, M. M.; Pereira, F. F.; E Zanuncio, J.C., 2011. Development and reproduction of Podisus nigrispinus (Hemiptera: Pentatomidae) fed with Thyrinteina arnobia (Lepidoptera: Geometridae) reared on guava leaves. Brazilian Archives of Biology and Technology, $54,429-434$

Pastori, P. L.; Pereira, F. F.; Andrade, G. S.; Silva, R. O.; Zanuncio, J. C.; Pereira, A. I. A., 2012. Reproduction of Trichospilus diatraeae (Hymenoptera: Eulophidae) in pupae of two lepidopterans defoliators of eucalypt. Revista Colombiana de Entomologia, 38, 91-93.

Pereira, L. G. B. A., 2007. Lagarta-Parda, Thyrinteina arnobia, principal lepidóptero desfolhador da cultura do eucalipto. Belo Horizonte, Minas Gerais: Fundação Centro Tecnológico de Minas Gerais CETEC, p.29. (Dossiê Técnico).

Rossi-Zalaf, L. S.; Alves, S. B.; Lopes, R. B.; Silveira Neto, S.; Tanzini, M. R., 2008. Interação de micro-organismos com outros agentes de controle de pragas e doenças. In; Alves, S. B; Lopes, R. B. (Eds.). Controle microbiano de pragas na América Latina: Avanços e Desafios. Piracicaba-SP: FEALQ. pp. 279-302.

Silva, R. Z. D.; Neves, P. M. D. O. J.; Santoro, P. H., 2005. Técnicas e parâmetros utilizados nos estudos de compatibilidade entre fungos entomopatogênicos e produto fitossanitários Semina: Ciências Biológicas e da Saúde, 26, 305-312.

Sosa-Gomez, D. R. 2005. Seletividade de agroquímicos para fungos entomopatogênicos. Londrina-PR: Embrapa Soja.

Souza, N. M; Junqueira, L. R; Wilcken, C. F; Soliman, E. P; Camargo, M. B.; Nickele, M. P; Barbosa, L. R. Ressurgência de uma antiga ameaça: Gorgulho-do-eucalipto Gonipterus platensis (Coleoptera: Curculionidae). Piracicaba-SP: Instituto de Pesquisas e Estudos Florestais - IPEF, 2016. 20 p. (Circular Técnica, 209).

Wilcken, C. F. Percevejo bronzeado do eucalipto (Thaumastocoris peregrinus) (Hemiptera: Thaumastocoridae): ameaça às florestas de eucalipto brasileiras. Botucatu-SP: Instituto de Pesquisas e Estudos Florestais - IPEF, 2008. 11 p.

Zappelini, L. O.; Almeida, J. E. M.; Gassen, M. H., 2005. Compatibilidade de fungos entomopatogênicos com emulsificantes para óleo vegetal e pó molhável. Arquivos do Instituto Biológico, 72, 1-63.

Zimmermann, G., 1975. Über die Wirkung systemischer Fungizide auf verschiedene insektenpathogene Fungi imperfecti in vitro. Nachrichtenblatt des Deutschen Pflanzenschutdienstes, 27,113-117. 